\title{
An Interpolation Problem for Hilbert-Schmidt Operator-Valued Stationary Processes
}

\author{
L. Klotz
}

\begin{abstract}
The paper contains a solution of the following interpolation problem for HilbertSchmidt operator-valued stationary processes on the real line: Assume that the values of the process on the integers are known. Determine the best linear approximation of an unknown value on the basis of the known values and compute the approximation error. Our results generalize previous results of Yaglom and Salehi for univariate and $q$-variate processes, respectively.
\end{abstract}

Keywords: Hilbert-Schmidt operator-valued stationary processes, linear interpolation

AMS subject classification: Primary 60 G 25, 60 G 10, secondary 47 B 10

\section{Introduction}

Let $\mathbf{x}$ be a univariate (weakly) stationary process on the real line $\mathbb{R}$. Assume that the values $x_{k}$, where $k$ runs through the set $\mathbb{Z}$ of all integers, are known. A. M. Yaglom [9] studied the problem to determine the error of the best linear interpolation of a value $x_{s} \quad(s \in \mathbb{R})$ by the known values. Using Kolmogorov's isomorphism between time and spectral domains of a stationary process, he transferred the problem to the spectral domain of $\mathbf{x}$ and solved it with the aid of elementary Hilbert space geometry. In [8 : Theorem 3.2] H. Salehi extended Yaglom's result to $q$-variate $(q<\infty)$ stationary processes and additionally gave the explicit form of the best linear interpolation.

The present paper is devoted to the solution of Yaglom's interpolation problem for Hilbert-Schmidt operator-valued stationary processes. Since the class of these processes can be identified with the class of infinite-variate stationary processes (cf. [6: pp. 346 347]), our assertions can be regarded as generalizations of Yaglom's and Salehi's results.

Our considerations are based on some facts on Hilbert-Schmidt and trace class operators, which are briefly summarized in Section 2. A precise formulation of Yaglom's interpolation problem transferred to the spectral domain is given in Section 3. The concluding Section 4 contains its solution. Starting from the spectral measure of the process, we construct a certain function $\Phi_{s}$ (see formula (4.4)) and show that this function can be taken for the best linear interpolation. One step of our proof is the computation of the interpolation error (see Theorem 4.8).

L. Klotz: Math. Inst. der Univ., PF 1009 20, D-04009 Leipzig 
We make use of the following notations. The symbol $\mathbb{N}$ stands for the set of positive integers. For a subset $A$ of $\mathbb{R}$ and a real number $r$, let $A+r$ be the shifted set $A+r=$ $\{a+r: a \in A\}$. The range of a linear operator $X$ is denoted by $\mathcal{R}(X)$. If $X$ is closable, then $X^{\sim}$ is its closure, if $X$ is densely defined, then $X^{*}$ is its adjoint. The identity operator (in a certain Hilbert space) is denoted by $I$. The symbols $\sum_{k}$ and $\sum_{k}^{\prime}$ mean that the summation index runs through $\mathbb{Z}$ and any finite subset of $\mathbb{Z}$, respectively.

\section{Preliminaries from operator theory}

For the reader's convenience we recall some facts on linear operators in Hilbert spaces. If we say that a certain property exists weakly or strongly, this means throughout this paper that the property exists with respect to weak or strong topologies, respectively, of the underlying Hilbert spaces.

Lemma 2.1 (cf. [7: Theorem 13.2]). Let $X, Y$ and $X Y$ be densely defined linear operators in a Hilbert space. Then $(X Y)^{*}$ is an extension of $Y^{*} X^{*}$. If, additionally, $X$ is bounded, then $(X Y)^{*}=Y^{*} X^{*}$.

Let $\mathcal{K}$ be a separable Hilbert space with inner product $(\cdot, \cdot)_{\mathcal{K}}$ and norm $|\cdot|_{\mathcal{K}}$ over the field of complex numbers $\mathbb{C}$. Let $\mathcal{S}^{+}$be the set of non-negative selfadjoint operators in $\mathcal{K}, \mathcal{B}$ the Banach algebra of bounded linear operators, $\mathfrak{S}_{2}$ the Hilbert space of HilbertSchmidt operators, and $\mathfrak{S}_{1}$ the norm ideal of trace class operators on $\mathcal{K}$. The norms of $\mathcal{B}, \mathfrak{S}_{2}$, and $\mathfrak{S}_{1}$ are denoted by $|\cdot|,|\cdot|_{2}$, and $|\cdot|_{1}$, respectively. Set $\mathcal{B}^{+}=B \cap \mathcal{S}^{+}$and $\mathfrak{S}_{1}^{+}=\mathfrak{S}_{1} \cap \mathcal{S}^{+}$. For $X, Y \in \mathcal{B}^{+}$, we write $Y \geq X$ if $Y-X \in \mathcal{B}^{+}$. The non-negative selfadjoint square root of an operator $X$ of $\mathcal{S}^{+}$is denoted by $X^{\frac{1}{2}}$.

If $X \in \mathcal{B}$, then the symbol $X^{\#}$ stands for the generalized inverse of $X$, i.e. $X^{\#} u=0$ if $u$ is orthogonal to $\mathcal{R}(X)$ and $X^{\#} u=v$ if $u \in \mathcal{R}(X)$. Here $v$ denotes the unique element of the orthogonal complement of the kernel of $X$ such that $X v=u$ (cf. [5: Definition $2.11])$.

Lemma 2.2. Let $X, Y \in \mathcal{B}^{+}$and $Y \geq X$. Then:

(i) $\mathcal{R}\left(Y^{\frac{1}{2}}\right) \supseteq \mathcal{R}\left(X^{\frac{1}{2}}\right)$.

(ii) $\left(X^{\frac{1}{2}} Y^{\# \frac{1}{2}}\right) \sim$ and $Y^{\# \frac{1}{2}} X^{\frac{1}{2}}$ belong to $\mathcal{B}$.

(iii) $\left|\left(X^{\frac{1}{2}} Y^{\# \frac{1}{2}}\right) \sim\right|=\left|Y^{\# \frac{1}{2}} X^{\frac{1}{2}}\right| \leq 1$.

Proof. Note first that $\left(X^{\frac{1}{2}} Y^{\# \frac{1}{2}}\right)^{*}=Y^{\# \frac{1}{2}} X^{\frac{1}{2}}$ by Lemma 2.1. For $u \in \mathcal{R}\left(Y^{\frac{1}{2}}\right)$, we have

$$
\begin{aligned}
\left|X^{\frac{1}{2}} Y^{\# \frac{1}{2}} u\right|_{\mathcal{K}}^{2} & =\left(\left(X^{\frac{1}{2}} Y^{\# \frac{1}{2}}\right)^{*} X^{\frac{1}{2}} Y^{\# \frac{1}{2}} u, u\right)_{\mathcal{K}} \\
& =\left(Y^{\# \frac{1}{2}} X Y^{\# \frac{1}{2}} u, u\right)_{\mathcal{K}} \\
& \leq\left(Y^{\# \frac{1}{2}} Y Y^{\# \frac{1}{2}} u, u\right)_{\mathcal{K}} \\
& =|u|_{\mathcal{K}}^{2} .
\end{aligned}
$$

Thus statements (ii) and (iii) follow.

The relation $Y \geq X$ implies that the kernel of $Y^{\frac{1}{2}}$ is included in the kernel of $X^{\frac{1}{2}}$. Hence the closure of $\mathcal{R}\left(Y^{\frac{1}{2}}\right)$ contains the closure of $\mathcal{R}\left(X^{\frac{1}{2}}\right)$. Since $Y^{\# \frac{1}{2}} X^{\frac{1}{2}} \in \mathcal{B}$, we obtain also statement (i) 
A detailed study of the symmetric norm ideals $\mathfrak{S}_{1}$ and $\mathfrak{S}_{2}$ can be found, e.g., in [1: Chapter III]. Here we summarize some results needed in the sequel.

Let $\operatorname{tr}$ denote the trace of a trace class operator. Then

$$
\begin{cases}|X|_{1}=\operatorname{tr}\left(X X^{*}\right)^{\frac{1}{2}} & \text { if } X \in \mathfrak{S}_{1} \\ |Y|_{2}^{2}=\operatorname{tr}\left(Y Y^{*}\right) & \text { if } Y \in \mathfrak{S}_{2} .\end{cases}
$$

It follows that if $X \in \mathfrak{S}_{1}^{+}$, then $X^{\frac{1}{2}} \in \mathfrak{S}_{2}$ and $|X|_{1}=\left|X^{\frac{1}{2}}\right|_{2}^{2}$. Furthermore, if $X, Y \in \mathfrak{S}_{2}$, then $X Y \in \mathfrak{S}_{1}$ and

$$
|\operatorname{tr}(X Y)| \leq|X Y|_{1} \leq|X|_{2}|Y|_{2}
$$

Lemma 2.3. Let $\left\{X_{n}\right\}_{n \in \mathbb{N}} \subseteq \mathfrak{S}_{1}^{+}$be an increasing sequence (i.e. $X_{n+1} \geq X_{n} n \in$ $\mathbb{N})$ converging weakly to an operator $X$ of $\mathfrak{S}_{1}$. Then $\left\{\operatorname{tr} X_{n}\right\}_{n \in \mathbb{N}}$ is an increasing sequence converging to $\operatorname{tr} X$.

Proof. Let $\left\{u_{k}\right\}_{k \in \mathbb{N}}$ be an orthonormal basis of $\mathcal{K}$. Then the sequence $\left\{\operatorname{tr} X_{n}\right\}_{n \in \mathbb{N}}=$ $\left\{\sum_{k=1}^{\infty}\left(X_{n} u_{k}, u_{k}\right)_{\mathcal{K}}\right\}_{n \in \mathbb{N}}$ increases and

$$
\lim _{n \rightarrow \infty} \operatorname{tr} X_{n}=\lim _{n \rightarrow \infty} \sum_{k=1}^{\infty}\left(X_{n} u_{k}, u_{k}\right)_{\mathcal{K}}=\sum_{k=1}^{\infty}\left(X u_{k}, u_{k}\right)_{\mathcal{K}}=\operatorname{tr} X
$$

Thus the statement is proved

Lemma 2.4 (cf. [2: Korollar 5 and Section 6] and [4: Lemma 4]). Let $X, Z \in \mathfrak{S}_{1}^{+}$ and $Y \in \mathcal{B}$. Assume that $\left(\begin{array}{cc}X & Y \\ Y^{*} Z\end{array}\right)$ is a non-negative selfadjoint operator on $\mathcal{K} \oplus \mathcal{K}$. Then:

(i) $Y Z^{\# \frac{1}{2}}$ and $X^{\# \frac{1}{2}} Y Z^{\# \frac{1}{2}}$ are densely defined and bounded.

(ii) $\left(Y Z^{\# \frac{1}{2}}\right)^{\sim} \in \mathfrak{S}_{2}$.

(iii) $\left|\left(X^{\# \frac{1}{2}} Y Z^{\# \frac{1}{2}}\right) \sim\right| \leq 1$.

(iv) $X \geq\left(Y Z^{\# \frac{1}{2}}\right)^{\sim}\left(Y Z^{\# \frac{1}{2}}\right)^{*}$.

Finally, we remark that for $\mathfrak{S}_{1^{-}}$or $\mathfrak{S}_{2}$-valued functions the notions of weak, strong, and Bochner measurability coincide (see [3: Lemma 5]). In this case we simply speak of measurable functions.

\section{Yaglom's interpolation problem for $\mathfrak{S}_{2}(\mathcal{K}, \mathcal{H})$-valued stationary processes}

Let $\mathcal{H}$ be a Hilbert space over $\mathbb{C}$ and $\mathfrak{S}_{2}(\mathcal{K}, \mathcal{H})$ the space of Hilbert-Schmidt operators of $\mathcal{K}$ to $\mathcal{H}$. A weakly continuous map $\mathbf{X}: \mathbb{R} \ni t \rightarrow X_{t} \in \mathfrak{S}_{2}(\mathcal{K}, \mathcal{H})$ is called an $\mathfrak{S}_{2}(\mathcal{K}, \mathcal{H})$-valued stationary process if the function $\mathbb{R} \times \mathbb{R} \ni(s, t) \rightarrow X_{t}^{*} X_{s}$ depends only on $s-t$.

For $t \in \mathbb{R}$, denote by $e_{t}$ the function $e_{t}(\lambda)=e^{i t \lambda}$ and by $E_{t}$ the function $E_{t}(\lambda)=$ $e_{t}(\lambda) I \quad(\lambda \in \mathbb{R})$. Let $E_{0}=: E$. There exists a unique $\mathfrak{S}_{1}^{+}$-valued Borel measure $F$ on $\mathbb{R}$ such that $X_{0}^{*} X_{t}=\int_{\mathbb{R}} e_{t}(\lambda) F(d \lambda) \quad(t \in \mathbb{R})$ (we remark that the integration variable will often be omitted in the notation of integrals). The measure $F$ is absolutely continuous 
with respect to the non-negative finite Borel measure $\tau=\operatorname{tr} F$. Since the Banach space $\mathfrak{S}_{1}$ has the Radon-Nikodym property, there exists the Radon-Nikodym derivative $\frac{d F}{d \tau}$, which will be denoted by $G$. It should be mentioned that the results of our paper remain true if $\tau$ is replaced by any non-negative $\sigma$-finite Borel measure on $\mathbb{R}$ (cf. [5: Lemma 4.5]). The function $G$ is a measurable function, whose values belong to $\mathfrak{S}_{1}^{+} \tau$-a.e. In the following we will not emphasize each time that a certain property of a measurable function is to be understood as a property that holds true a.e. with respect to the underlying non-negative measure.

Let $\mathcal{A}$ be the set of (not necessarily densely defined and not necessarily bounded) linear operators in $\mathcal{K}$. By $L^{2}(F)$ we denote the Hilbert space of (equivalence classes of) $\mathcal{A}$-valued functions $\Phi$ on $\mathbb{R}$ such that $\Phi G^{\frac{1}{2}}$ is a measurable $\mathfrak{S}_{2}$-valued function and $|\Phi|_{F}^{2}:=\int_{\mathbb{R}}\left|\Phi G^{\frac{1}{2}}\right|_{2}^{2} d \tau<\infty$ (cf. [5: Definition 4.8 and formula (4.10)] and [3: Lemma 5 and Section 3]). As usual we will not work with equivalence classes but with their representatives, i.e. with functions.

A $\mathcal{B}$-valued function $T$ on $\mathbb{R}$ is called a trigonometric polynomial if it is of the form $T=\sum_{k}^{\prime} Y_{k} e_{k}$, with coefficients $Y_{k}$ from $\mathcal{B}$. Clearly, trigonometric polynomials are $2 \pi-$ periodic functions and belong to $L^{2}(F)$. The linear set of all trigonometric polynomials is denoted by $\mathcal{T}$ and its closure in $L^{2}(F)$ by $\overline{\mathcal{T}}$.

Let $s \in \mathbb{R}$. Yaglom's interpolation problem for the $\mathfrak{S}_{2}(\mathcal{K}, \mathcal{H})$-valued stationary process $\mathbf{X}$ consists in determining the best linear interpolation of $X_{s}$ as well as the interpolation error on the basis of the known values $X_{k} \quad(k \in \mathbb{Z})$. Using Kolmogorov's isomorphism between the space spanned by the values of the process and the space $L^{2}(F)$ (cf. [5: Theorem 7.8]), we can give the problem an equivalent form: Find the orthogonal projection in $L^{2}(F)$ of the function $E_{s}$ onto $\overline{\mathcal{T}}$ as well as the distance $\delta_{s}$ of $E_{s}$ to $\overline{\mathcal{T}}$. This is the form we will study in the next section.

\section{Best linear interpolation and interpolation error}

For $k \in \mathbb{Z}$, let $J_{k}$ be the interval $[2 k \pi, 2(k+1) \pi)$. Let $J_{0}=: J$ and let $\mathfrak{B}$ be the Borel $\sigma$-algebra of $J$. For $k \in \mathbb{Z}$ and $B \in \mathfrak{B}$ set

$$
\left.\begin{array}{rl}
F_{k}(B) & =F(B+2 k \pi) \\
\tau_{k}(B) & =\operatorname{tr} F_{k}(B) \\
\mu(B) & =\sum_{j} \tau_{j}(B) \\
M(B) & =\sum_{j} F_{j}(B)
\end{array}\right\} .
$$

Note that series $(4.1)_{4}$ converges in $\mathfrak{S}_{1}$ and that $M$ is an $\mathfrak{S}_{1}^{+}$-valued measure on $\mathfrak{B}$.

Denote by $G_{k}$ the Radon-Nikodym derivative $\frac{d F_{k}}{d \mu} \quad(k \in \mathbb{Z})$ and by $N$ the RadonNikodym derivative $\frac{d M}{d \mu}$. Then $N$ is an $\mathfrak{S}_{1}^{+}$-valued measurable function, $N=\sum_{j} G_{j}$, and

$$
\int_{J}|N|_{1} d \mu<\infty
$$

Lemma 4.1. The restrictions of the trigonometric polynomials to $J$ are dense in $L^{2}(M)$. 
Proof. Assume that a function $\Psi$ of $L^{2}(M)$ satisfies $\operatorname{tr} \int_{J} \Psi N^{\frac{1}{2}}\left(e_{n} Y N^{\frac{1}{2}}\right)^{*} d \mu=0$ for all $Y \in \mathcal{B}$ and $n \in \mathbb{Z}$. Then $\int_{J} \Psi N e_{n} d \mu=0$ for all $n \in \mathbb{Z}$, hence $\Psi N=0$ and also $\Psi N^{\frac{1}{2}}=\left(\Psi N N^{\# \frac{1}{2}}\right)^{\sim}=0 \mu$-a.e., which yields $\Psi=0$ in $L^{2}(M)$

For an $\mathcal{A}$-valued function $\Phi$ on $\mathbb{R}$, set $\hat{\Phi}_{k}(\lambda)=\Phi(\lambda+2 k \pi)(\lambda \in J, k \in \mathbb{Z})$. Note that $\hat{\Phi}_{0}$ is the restriction of $\Phi$ to $J$ and that if $\Phi$ is $2 \pi$-periodic, the functions $\hat{\Phi}_{k} \quad(k \in \mathbb{Z})$ coincide with $\hat{\Phi}_{0}$.

Lemma 4.2. If $\Phi$ is an $\mathcal{A}$-valued function on $\mathbb{R}$ such that $\Phi G^{\frac{1}{2}}$ is measurable and $\mathfrak{S}_{2}$-valued $\tau$-a.e., then

$$
\int_{\mathbb{R}}\left|\Phi G^{\frac{1}{2}}\right|_{2}^{2} d \tau=\sum_{k} \int_{J}\left|\hat{\Phi}_{k} G_{k}^{\frac{1}{2}}\right|_{2}^{2} d \mu .
$$

Proof. A straightforward computation yields

$$
\begin{aligned}
\int_{\mathbb{R}}\left|\Phi G^{\frac{1}{2}}\right|_{2}^{2} d \tau & =\sum_{k} \int_{J_{k}}\left|\Phi G^{\frac{1}{2}}\right|_{2}^{2} d \tau \\
& =\sum_{k} \int_{J}\left|\Phi(\lambda+2 k \pi) G(\lambda+2 k \pi)^{\frac{1}{2}}\right|_{2}^{2} \tau(d \lambda+2 k \pi) \\
& =\sum_{k} \int_{J}\left|\hat{\Phi}_{k}\left(\frac{d F_{k}}{d \tau_{k}}\right)^{\frac{1}{2}}\right|_{2}^{2} d \tau_{k} \\
& =\sum_{k} \int_{J}\left|\hat{\Phi}_{k}\left(\frac{d F_{k}}{d \tau_{k}}\right)^{\frac{1}{2}}\right|_{2}^{2} \frac{d \tau_{k}}{d \mu} d \mu \\
& =\sum_{k} \int_{J}\left|\hat{\Phi}_{k} G_{k}^{\frac{1}{2}}\right|_{2}^{2} d \mu
\end{aligned}
$$

and the statement is proved

Lemma 4.3. Let $\Phi \in L^{2}(F)$. Then:

(i) $\int_{J} \sum_{k}\left|\hat{\Phi}_{k} G_{k}^{\frac{1}{2}}\right|_{2}^{2} d \mu=|\Phi|_{F}^{2}$.

(ii) $\sum_{k}\left|\hat{\Phi}_{k} G_{k}^{\frac{1}{2}}\right|_{2}^{2}<\infty \mu$-a.e.

(iii) $\sum_{k}\left|\hat{\Phi}_{k} G_{k}\right|_{1}<\infty \mu$-a.e.

(iv) $\sum_{k} \hat{\Phi}_{k} G_{k}$ converges in $\mathfrak{S}_{1} \mu$-a.e.

Proof. Assertions (i) and (ii) are immediate consequences of B. Levi's theorem and Lemma 4.2. Using (2.1), Cauchy's inequality and (ii), we obtain statement (iii) and, hence, statement (iv)

For $s \in \mathbb{R}$, set

$$
W_{s}(\lambda)=\sum_{k} e_{2 k \pi}(s) G_{k}(\lambda) \quad(\lambda \in J) .
$$

Note that Lemma 4.3 implies the $\mu$-a.e. convergence of the series (4.3) in $\mathfrak{S}_{1}$. Thus, $W_{s}$ is an $\mathfrak{S}_{1}$-valued measurable function. 
Lemma 4.4. Let $s \in \mathbb{R}, \lambda \in J$, and let $V_{s}(\lambda)$ be of the form $\sum_{k}^{\prime} e_{2 k \pi}(s) G_{k}(\lambda)$ or equal to $W_{s}(\lambda)$. Then:

(i) $V_{s}(\lambda) N^{\#}(\lambda)^{\frac{1}{2}}$ and $N^{\#}(\lambda)^{\frac{1}{2}} V_{s}(\lambda) N^{\#}(\lambda)^{\frac{1}{2}}$ are densely defined and bounded.

(ii) $\left(V_{s}(\lambda) N^{\#}(\lambda)^{\frac{1}{2}}\right)^{\sim} \in \mathfrak{S}_{2}$.

(iii) $\left|\left(N^{\#}(\lambda)^{\frac{1}{2}} V_{s}(\lambda) N^{\#}(\lambda)^{\frac{1}{2}}\right)^{\sim}\right| \leq 1$.

(iv) $N(\lambda) \geq\left(V_{s}(\lambda) N^{\#}(\lambda)^{\frac{1}{2}}\right)^{\sim}\left(V_{s}(\lambda) N^{\#}(\lambda)^{\frac{1}{2}}\right)^{*}$.

Proof. Since $\left(\begin{array}{cc}N(\lambda) & V_{s}(\lambda)^{*} \\ V_{s}(\lambda) * & N(\lambda)\end{array}\right)$ is a non-negative selfadjoint operator on $\mathcal{K} \oplus \mathcal{K}$, the results immediately follow from Lemma 2.4

For $s \in \mathbb{R}$, define the $2 \pi$-periodic function $\Phi_{s}$ by

$$
\Phi_{s}(\lambda)=e_{s}(\lambda-2 k \pi)\left[W_{s}(\lambda-2 k \pi) N^{\#}(\lambda-2 k \pi)^{\frac{1}{2}}\right]^{\sim} N^{\#}(\lambda-2 k \pi)^{\frac{1}{2}}
$$

for $\lambda \in J_{k}$ and $k \in \mathbb{Z}$. Now our goal is to prove that $\Phi_{s}$ is the orthogonal projection in $L^{2}(F)$ of the function $E_{s}$ onto $\overline{\mathcal{T}}$. To do this, we first show that $\Phi_{s}$ belongs to $\overline{\mathcal{T}}$. Then we compute the interpolation error $\delta_{s}$ and the distance $\left|E_{s}-\Phi_{s}\right|_{F}$, which turn out to be equal.

Lemma 4.5. For $\tau$-a.a. $\lambda \in \mathbb{R}$, the operator $\Phi_{s}(\lambda) G(\lambda)^{\frac{1}{2}}$ can be defined and belongs to $\mathfrak{S}_{2}$. The function $\Phi_{s} G^{\frac{1}{2}}$ is measurable.

Proof. For $k \in \mathbb{Z}$, set $A_{k}=\left\{\lambda \in J_{k}: \frac{d \tau_{k}}{d \mu}(\lambda-2 k \pi)=0\right\}$. Then

$$
\tau\left(A_{k}\right)=\int_{A_{k}} \frac{d \tau_{k}}{d \mu}(\lambda-2 k \pi) \mu(d \lambda-2 k \pi)=0,
$$

and if $\lambda \in J_{k} \backslash A_{k}$, we have

$$
\begin{aligned}
G_{k}(\lambda-2 k \pi) & =\frac{d F_{k}}{d \mu}(\lambda-2 k \pi) \\
& =\frac{d F_{k}}{d \tau_{k}}(\lambda-2 k \pi) \frac{d \tau_{k}}{d \mu}(\lambda-2 k \pi) \\
& =G(\lambda) \frac{d \tau_{k}}{d \mu}(\lambda-2 k \pi) .
\end{aligned}
$$

Since $N \geq G_{k}$, the first part of the lemma follows from Lemma 2.2 and Lemma 4.4. The measurability of $\Phi_{s} G^{\frac{1}{2}}$ is a consequence of [3: Corollary 2]

Lemma 4.6. If $T \in \mathcal{T}$, then

$$
\int_{\mathbb{R}}\left|\left(\Phi_{s}-T\right) G^{\frac{1}{2}}\right|_{2}^{2} d \tau=\int_{J}\left|\left(\Phi_{s}-T\right) N^{\frac{1}{2}}\right|_{2}^{2} d \mu
$$

Proof. Using the relation $N \geq G_{k}$ and Lemma 2.2, we can write

$$
\begin{aligned}
\left|\left(\Phi_{s}-T\right) G_{k}^{\frac{1}{2}}\right|_{2}^{2} & =\operatorname{tr}\left(\left(\Phi_{s}-T\right) G_{k}^{\frac{1}{2}}\left[\left(\Phi_{s}-T\right) G_{k}^{\frac{1}{2}}\right]^{*}\right) \\
& =\operatorname{tr}\left(\left(\Phi_{s}-T\right) N^{\frac{1}{2}} N^{\# \frac{1}{2}} G_{k}^{\frac{1}{2}}\left[\left(\Phi_{s}-T\right) G_{k}^{\frac{1}{2}}\right]^{*}\right) \quad(k \in \mathbb{Z}) . \\
& =\operatorname{tr}\left(\left(\Phi_{s}-T\right) N^{\frac{1}{2}}\left[\left(\Phi_{s}-T\right) G_{k}^{\frac{1}{2}}\left(N^{\# \frac{1}{2}} G_{k}^{\frac{1}{2}}\right)^{*}\right]^{*}\right)
\end{aligned}
$$


From Lemma 2.1 it follows that

$$
\left(\Phi_{s}-T\right) G_{k}^{\frac{1}{2}}\left(N^{\# \frac{1}{2}} G_{k}^{\frac{1}{2}}\right)^{*} \text { is an extension of }\left(\Phi_{s}-T\right) G_{k} N^{\# \frac{1}{2}},
$$

therefore

$$
\left(\left(\Phi_{s}-T\right) G_{k} N^{\# \frac{1}{2}}\right)^{*} \quad \text { is an extension of }\left(\left(\Phi_{s}-T\right) G_{k}^{\frac{1}{2}}\left(N^{\# \frac{1}{2}} G_{k}^{\frac{1}{2}}\right)^{*}\right)^{*} .
$$

Since

$$
\left(\left(\Phi_{s}-T\right) G_{k}^{\frac{1}{2}}\left(N^{\# \frac{1}{2}} G_{k}^{\frac{1}{2}}\right)^{*}\right)^{*} \in \mathcal{B},
$$

we have, in fact,

$$
\left(\left(\Phi_{s}-T\right) G_{k} N^{\# \frac{1}{2}}\right)^{*}=\left(\left(\Phi_{s}-T\right) G_{k}^{\frac{1}{2}}\left(N^{\# \frac{1}{2}} G_{k}^{\frac{1}{2}}\right)^{*}\right)^{*} \quad(k \in \mathbb{Z}) .
$$

A simple computation gives

$$
\begin{aligned}
\left(\Phi_{s}-\right. & T) N^{\frac{1}{2}}\left(\left(\Phi_{s}-T\right) G_{k} N^{\# \frac{1}{2}}\right)^{*} \\
= & \left(e_{s}\left(W_{s} N^{\# \frac{1}{2}}\right)^{\sim}-T N^{\frac{1}{2}}\right) \\
& \times\left(\left(N^{\# \frac{1}{2}} G_{k} N^{\# \frac{1}{2}}\right)^{\sim}\left(e_{s} W_{s} N^{\# \frac{1}{2}}\right)^{*}-\left(G_{k} N^{\# \frac{1}{2}}\right)^{*} T^{*}\right)
\end{aligned}
$$

Using Lemma 2.1, the relation $N \geq G_{k}$ and Lemma 2.2, we get

$$
\begin{aligned}
\left(G_{k} N^{\# \frac{1}{2}}\right)^{*} T^{*} & =N^{\# \frac{1}{2}} G_{k} T^{*} \\
& =N^{\# \frac{1}{2}} G_{k} N^{\# \frac{1}{2}} N^{\frac{1}{2}} T^{*} \quad(k \in \mathbb{Z}) . \\
& =N^{\# \frac{1}{2}} G_{k} N^{\# \frac{1}{2}}\left(T N^{\frac{1}{2}}\right)^{*}
\end{aligned}
$$

Now, combining (4.5) - (4.8), we obtain

$$
\begin{aligned}
& \left|\left(\Phi_{s}-T\right) G_{k}^{\frac{1}{2}}\right|_{2}^{2} \\
& \quad=\operatorname{tr}\left(\left[e_{s}\left(W_{s} N^{\# \frac{1}{2}}\right)^{\sim}-T N^{\frac{1}{2}}\right]\left(N^{\# \frac{1}{2}} G_{k} N^{\# \frac{1}{2}}\right)^{\sim}\left[e_{s}\left(W_{s} N^{\# \frac{1}{2}}\right)^{\sim}-T N^{\frac{1}{2}}\right]^{*}\right)
\end{aligned}
$$

for all $k \in \mathbb{Z}$. If $u, v \in \mathcal{R}\left(N^{\frac{1}{2}}\right)$, then

$$
\sum_{k}\left(N^{\# \frac{1}{2}} G_{k} N^{\# \frac{1}{2}} u, v\right)_{\mathcal{K}}=(u, v)_{\mathcal{K}} .
$$

Taking into account Lemma 4.4/(iii), we can easily derive that $\sum_{k}\left(N^{\# \frac{1}{2}} G_{k} N^{\# \frac{1}{2}}\right)^{\sim}=$ $I$ with respect to the weak topology. Then from (4.10), Lemma 2.3, the monotone convergence theorem, and (4.4) it follows

$$
\begin{aligned}
\sum_{k} & \int_{J}\left|\left(\Phi_{s}-T\right) G_{k}^{\frac{1}{2}}\right|_{2}^{2} d \mu \\
& =\int_{J} \operatorname{tr}\left(\left[e_{s}\left(W_{s} N^{\# \frac{1}{2}}\right)^{\sim}-T N^{\frac{1}{2}}\right]\left[e_{s}\left(W_{s} N^{\# \frac{1}{2}}\right)^{\sim}-T N^{\frac{1}{2}}\right]^{*}\right) d \mu \\
& =\int_{J}\left|\left(\Phi_{s}-T\right) N^{\frac{1}{2}}\right|_{2}^{2} d \mu .
\end{aligned}
$$

Since Lemma 4.2 yields

$$
\int_{\mathbb{R}}\left|(\Phi-T) G^{\frac{1}{2}}\right|_{2}^{2} d \tau=\sum_{k} \int_{J}\left|\left(\Phi_{s}-T\right) G_{k}^{\frac{1}{2}}\right|_{2}^{2} d \mu,
$$

the desired result can be concluded from (4.11) 
Proposition 4.7. For $s \in \mathbb{R}$, the function $\Phi_{s}$ is an element of $\overline{\mathcal{T}}$ and its restriction to $J$ belongs to $L^{2}(M)$.

Proof. By (4.4), Lemma 4.4, and (4.2),

$$
\int_{J}\left|\Phi_{s} N^{\frac{1}{2}}\right|_{2}^{2} d \mu=\int_{J}\left|e_{s}\left(W_{s} N^{\# \frac{1}{2}}\right)^{\sim}\right|_{2}^{2} d \mu \leq \int_{J}|N|_{1} d \mu<\infty .
$$

Therefore, the restriction of $\Phi_{s}$ to $J$ belongs to $L^{2}(M)$. An appeal to Lemmas 4.6 and 4.1 completes the proof

Theorem 4.8. For $s \in \mathbb{R}$, the square $\delta_{s}^{2}$ of the distance of $E_{s}$ to $\overline{\mathcal{T}}$ is equal to $\operatorname{tr} \int_{J}\left[N-\Phi_{s} N^{\frac{1}{2}}\left(\Phi_{s} N^{\frac{1}{2}}\right)^{*}\right] d \mu=|E|_{F}^{2}-\left|\Phi_{s}\right|_{F}^{2}$.

Proof. First note that for $T \in \mathcal{T}$,

$$
\begin{aligned}
\sum_{k} \mid & \left.\left(E-e_{-s} e_{2 k \pi}(-s) T\right) G_{k}^{\frac{1}{2}}\right|_{2} ^{2} \\
& =\sum_{k} \operatorname{tr}\left(G_{k}-T e_{-s} e_{2 k \pi}(-s) G_{k}-e_{s} e_{2 k \pi}(s) G_{k} T^{*}+T G_{k} T^{*}\right) \\
& =\operatorname{tr}\left(N-T\left(e_{s} W_{s}\right)^{*}-e_{s} W_{s} T^{*}+T N T^{*}\right)
\end{aligned}
$$

Relation (2.1) and Cauchy's inequality yield

$$
\left|\sum_{k}{ }^{\prime} \operatorname{tr}\left(e_{s} e_{2 k \pi}(s) G_{k} T^{*}\right)\right| \leq\left(\sum_{k}\left|G_{k}^{\frac{1}{2}}\right|_{2}^{2} \cdot \sum_{k}\left|T G_{k}^{\frac{1}{2}}\right|_{2}^{2}\right)^{\frac{1}{2}} .
$$

By Lemma 4.3, the function on the right-hand side of (4.13) is $\mu$-integrable. Therefore, (4.12) and the dominated convergence theorem imply

$$
\begin{aligned}
& \sum_{k} \int_{J}\left|\left[E-e_{-s} e_{2 k \pi}(-s) T\right] G_{k}^{\frac{1}{2}}\right|_{2}^{2} d \mu \\
& \quad=\operatorname{tr} \int_{J}\left[N-T\left(e_{s} W_{s}\right)^{*}-e_{s} W_{s} T^{*}+T N T^{*}\right] d \mu .
\end{aligned}
$$

According to Lemma 4.2, we have

$$
\left|E_{s}-T\right|_{F}^{2}=\sum_{k} \int_{J}\left|\left(E-e_{-s} e_{2 k \pi}(-s) T\right) G_{k}^{\frac{1}{2}}\right|_{2}^{2} d \mu
$$

Hence (4.14) gives

$$
\left|E_{s}-T\right|_{F}^{2}=\operatorname{tr} \int_{J}\left[N-T\left(e_{s} W_{s}\right)^{*}-e_{s} W_{s} T^{*}+T N T^{*}\right] d \mu .
$$


Now from (4.15), (4.4), Proposition 4.7, and Lemma 4.1 it follows

$$
\begin{aligned}
\delta_{s}^{2} & =\inf _{T \in \mathcal{T}}\left|E_{s}-T\right|_{F}^{2} \\
& =\inf _{T \in \mathcal{T}} \operatorname{tr} \int_{J}\left[N-T\left(e_{s} W_{s}\right)^{*}-e_{s} W_{s} T^{*}+T N T^{*}\right] d \mu \\
& =\inf _{T \in \mathcal{T}} \operatorname{tr} \int_{J}\left(N-\Phi_{s} N^{\frac{1}{2}}\left(\Phi_{s} N^{\frac{1}{2}}\right)^{*}+\left(T-\Phi_{s}\right) N^{\frac{1}{2}}\left[\left(T-\Phi_{s}\right) N^{\frac{1}{2}}\right]^{*}\right) d \mu \\
& =\int_{J}\left(N-\Phi_{s} N^{\frac{1}{2}}\left(\Phi_{s} N^{\frac{1}{2}}\right)^{*}\right) d \mu \\
& =|E|_{F}^{2}-\left|\Phi_{s}\right|_{F}^{2}
\end{aligned}
$$

and the statement is proved

Proposition 4.9. Let $s \in \mathbb{R}$. Then $\delta_{s}^{2}=\left|E_{s}-\Phi_{s}\right|^{2}$.

Proof. We have

$$
\begin{aligned}
\mid(E- & \left.e_{-s} e_{2 k \pi}(-s) \Phi_{s}\right)\left.G_{k}^{\frac{1}{2}}\right|_{2} ^{2} \\
= & \operatorname{tr}\left(G_{k}-\left(W_{s} N^{\# \frac{1}{2}}\right) \sim\left[G_{k} e_{2 k \pi}(s) N^{\# \frac{1}{2}}\right]^{*}\right. \\
& -N^{\frac{1}{2}}\left[e_{2 k \pi}(-s)\left(W_{s} N^{\# \frac{1}{2}}\right)^{\sim} N^{\# \frac{1}{2}} G_{k} N^{\# \frac{1}{2}}\right]^{*} \\
& \left.+\left(W_{s} N^{\# \frac{1}{2}}\right) \sim\left[\left(W_{s} N^{\# \frac{1}{2}}\right)^{\sim} N^{\# \frac{1}{2}} G_{k} N^{\# \frac{1}{2}}\right]^{*}\right)
\end{aligned}
$$

As in the proof of Lemma 4.6 it can be shown that

$$
\begin{gathered}
\sum_{k} \operatorname{tr} \int_{J}\left(\left(W_{s} N^{\# \frac{1}{2}}\right)^{\sim}\left[\left(W_{s} N^{\# \frac{1}{2}}\right)^{\sim} N^{\# \frac{1}{2}} G_{k} N^{\# \frac{1}{2}}\right]^{*}\right) d \mu \\
=\operatorname{tr} \int_{J} \Phi_{s} N^{\frac{1}{2}}\left(\Phi_{s} N^{\frac{1}{2}}\right)^{*} d \mu .
\end{gathered}
$$

For $u \in \mathcal{R}\left(N^{\frac{1}{2}}\right)$,

$$
\sum_{k} e_{2 k \pi}(s) G_{k} N^{\# \frac{1}{2}} u=W_{s} N^{\# \frac{1}{2}} u
$$

strongly. Since

$$
\left|\left(\sum_{k}{ }^{\prime} e_{2 k \pi}(s) G_{k} N^{\# \frac{1}{2}}\right)^{\sim}\right| \leq|N|^{\frac{1}{2}}
$$

by Lemma 4.4 , it is not hard to see that

$$
\sum_{k}\left[e_{2 k \pi}(s) G_{k} N^{\# \frac{1}{2}}\right]^{\sim}=\left(W_{s} N^{\# \frac{1}{2}}\right)^{\sim}
$$

with respect to the strong topology. Hence

$$
\sum_{k}\left(W_{s} N^{\# \frac{1}{2}}\right) \sim\left(e_{2 k \pi}(s) G_{k} N^{\# \frac{1}{2}}\right)^{*}=\left(W_{s} N^{\# \frac{1}{2}}\right) \sim\left(W_{s} N^{\# \frac{1}{2}}\right)^{*}
$$


weakly. On the other hand, Lemma 4.3 implies that

$$
\sum_{k}\left(W_{s} N^{\# \frac{1}{2}}\right)^{\sim}\left(e_{2 k \pi}(s) G_{k} N^{\# \frac{1}{2}}\right)^{*}=\sum_{k}\left(W_{s} N^{\# \frac{1}{2}}\right)^{\sim} e_{2 k \pi}(-s) N^{\# \frac{1}{2}} G_{k}
$$

converges in $\mathfrak{S}_{1}$. Therefore, from (4.18) we can conclude

$$
\sum_{k} \operatorname{tr}\left(\left(W_{s} N^{\# \frac{1}{2}}\right)^{\sim}\left[e_{2 k \pi}(s) G_{k} N^{\# \frac{1}{2}}\right]^{*}\right)=\operatorname{tr}\left[\left(W_{s} N^{\# \frac{1}{2}}\right)^{\sim}\left(W_{s} N^{\# \frac{1}{2}}\right)^{*}\right] .
$$

Analogously to the proof of (4.13) we obtain

$$
\begin{aligned}
& \left|\sum_{k}{ }^{\prime} \operatorname{tr}\left(\left(W_{s} N^{\# \frac{1}{2}}\right)^{\sim}\left[e_{2 k \pi}(s) G_{k} N^{\# \frac{1}{2}}\right]^{*}\right)\right| \\
& \quad \leq\left(\sum_{k}\left|\left(W_{s} N^{\# \frac{1}{2}}\right)^{\sim} N^{\# \frac{1}{2}} G_{k}^{\frac{1}{2}}\right|_{2}^{2} \sum_{k}\left|G_{k}^{\frac{1}{2}}\right|_{2}^{2}\right)^{\frac{1}{2}} .
\end{aligned}
$$

According to Lemma 4.3 and Proposition 4.7, the function on the right-hand side of (4.20) is $\mu$-integrable. Thus, (4.19), the dominated convergence theorem, and (4.4) give

$$
\begin{aligned}
\sum_{k} \operatorname{tr} \int_{J}\left(W_{s} N^{\# \frac{1}{2}}\right)^{\sim}\left[e_{2 k \pi}(s) G_{k} N^{\# \frac{1}{2}}\right]^{*} d \mu \\
=\operatorname{tr} \int_{J}\left(W_{s} N^{\# \frac{1}{2}}\right)^{\sim}\left(W_{s} N^{\# \frac{1}{2}}\right)^{*} d \mu \\
=\operatorname{tr} \int_{J} \Phi_{s} N^{\frac{1}{2}}\left(\Phi_{s} N^{\frac{1}{2}}\right)^{*} d \mu .
\end{aligned}
$$

Since

$$
N^{\frac{1}{2}}\left(e_{2 k \pi}(-s)\left(W_{s} N^{\# \frac{1}{2}}\right)^{\sim} N^{\# \frac{1}{2}} G_{k} N^{\# \frac{1}{2}}\right)^{*}=e_{2 k \pi}(s) N^{\# \frac{1}{2}} G_{k}\left(W_{s} N^{\# \frac{1}{2}}\right)^{*}
$$

for $k \in \mathbb{Z}$, in a similar way we obtain

$$
\begin{gathered}
\sum_{k} \operatorname{tr} \int_{J} N^{\frac{1}{2}}\left(e_{2 k \pi}(-s)\left(W_{s} N^{\# \frac{1}{2}}\right)^{\sim} N^{\# \frac{1}{2}} G_{k} N^{\# \frac{1}{2}}\right)^{*} d \mu \\
=\operatorname{tr} \int_{J} \Phi_{s} N^{\frac{1}{2}}\left(\Phi_{s} N^{\frac{1}{2}}\right)^{*} d \mu .
\end{gathered}
$$

Since, by Lemma 4.2,

$$
\left|E_{s}-\Phi_{s}\right|_{F}^{2}=\sum_{k} \int_{J}\left|\left[E-e_{-s} e_{2 k \pi}(-s) \Phi_{s}\right] G_{k}^{\frac{1}{2}}\right|_{2}^{2} d \mu,
$$

from (4.16), (4.17), (4.21) and (4.22)

$$
\left|E_{s}-\Phi_{s}\right|_{F}^{2}=\operatorname{tr} \int_{J}\left[N-\Phi_{s} N^{\frac{1}{2}}\left(\Phi_{s} N^{\frac{1}{2}}\right)^{*}\right] d \mu
$$

follows, and this coincides with $\delta_{s}^{2}$ by Theorem 4.8 
Combining Propositions 4.7 and 4.9, and Theorem 4.8, we immediately obtain the following main result of the present paper:

Theorem 4.10. For $s \in \mathbb{R}$, the orthogonal projection of $E_{s}$ onto $\overline{\mathcal{T}}$ is equal to the function $\Phi_{s}$ defined in (4.4).

Let us conclude with a remark on straightforward generalizations of our results.

Remark 4.11. The above results can be extended to $\mathfrak{S}_{2}(\mathcal{K}, \mathcal{H})$-valued homogeneous random fields on $\mathbb{R}^{n}, n \in \mathbb{N}$, in an obvious way. Moreover, using a transformation similar to that indicated in [9: p. 176] or [8: Proof of Theorem 3.3], we can solve an analogue of Yagloms interpolation problem for $\mathfrak{S}_{2}(\mathcal{K}, \mathcal{H})$-valued homogeneous fields on $\mathbb{Z}^{n}$. We omit the details.

\section{References}

[1] Gokhberg, I. Ts. and M. G. Krein: Introduction to the Theory of Linear Non-Selfadjoint Operators in Hilbert Space (in Russian). Moscow: Nauka 1965.

[2] Höschel, H.-P.: Über die Pseudoinverse eines zerlegten positiven linearen Operators. Math. Nachr. 74 (1976), $167-172$.

[3] Klotz, L.: Some Banach spaces of measurable operator-valued functions. Probab. Math. Statist. 12 (1991), $85-97$.

[4] Klotz, L.: Orthogonal projection and restricted subordination of Hilbert-Schmidt operatorvalued stationary processes. Z. Anal. Anw. 17 (1998), 557 - 563.

[5] Mandrekar, V. and H. Salehi: The square-integrability of operator-valued functions with respect to a non-negative operator-valued measure and the Kolmogorov isomorphism theorem. Indiana Univ. Math. J. 20 (1970), 545 - 563.

[6] Payen, R.: Fonctions aléatoires du second ordre à valeurs dans un espace de Hilbert. Ann. Inst. H. Poincaré Probab. Statist. 3 (1967), 323 - 396.

[7] Rudin, W.: Functional Analysis. New York et al.: McGraw-Hill 1973.

[8] Salehi, H.: On interpolation of q-variate stationary stochastic processes. Pacific J. Math. 28 (1969), 183 - 191.

[9] Yaglom, A. M.: On problems about the linear interpolation of stationary random sequences and processes (in Russian). Uspekhi Mat. Nauk 4 (1949)4, 173 - 178.

Received 06.04.2000 